\title{
Design and Performance of Space-Time Trellis Codes for Rapid Rayleigh Fading Channels
}

\author{
Salam A. Zummo and Saud A. Al-Semari
}

\begin{abstract}
Space-Time (ST) codes are known to provide high transmission rates, diversity and coding gains. In this paper, a tight upper bound on the error probability of ST codes over rapid fading channels is presented. Moreover, ST codes suitable for rapid fading channels are presented. These codes are designed using the QPSK and 16-QAM signal constellations. The proposed codes are based on two different encoding schemes. The first scheme uses a single trellis encoder, whereas the second scheme uses the I-Q encoding technique. Code design is achieved via partitioning the signal space such that the design criteria are maximized. As a solution for the decoding problem of I-Q ST codes, the paper introduces a low-complexity decoding algorithm. Results show that the I-Q ST codes using the proposed decoding algorithm outperform singleencoder ST codes with equal complexity. The proposed codes are tested over fading channels with different interleaving conditions, where it is shown that the new codes are robust under such imperfect interleaving conditions.
\end{abstract}

Index Terms: Fading channels, diversity, space-time codes, MIMO, I-Q, union bound, error probability.

\section{INTRODUCTION}

Wireless communication systems are emerging in various applications that require reliable communications at high data rates. Conventional channel coding techniques make use of the channel diversity to improve the performance. However, channels with memory suffer of insufficient channel diversity at the decoder, which degrades the performance severely. A popular channel model that exhibits memory is the block fading channel model $[1,2]$. This model approximates many wireless communication systems such as orthogonal frequency-division multiplexing (OFDM) and frequency-hopping (FH) systems. Recently, space-time (ST) codes [3-5] were proposed for block fading channels. ST codes combine efficiently transmit diversity and channel coding to provide reliable communications at high data rates. The performance and design criteria of ST codes were derived in [4] for quasi-static and rapid fading channels. In particular, the Chernoff bound was used to upper bound the pairwise error probability (PEP) which is a loose bound. Later, the exact expression for the PEP was derived in [6]. However, this expression cannot be computed using the transfer function (TF) of the trellis code. In this paper tight upper bounds on the PEP and the bit error probability (BEP) are derived for rapid fading

Manuscript received April 1, 2001

S. Zummo is with the Electrical Engineering and Computer Science Department at the University of Michigan, Ann Arbor, MI, USA, email: sazummo@umich.edu.

S. Al-Semari is with the Electrical Engineering Department at King Fahd University of Petroleum and Minerals (KFUPM), Dhahran, Saudi Arabia, email: semari@kfupm.edu.sa channels and evaluated using the TF of the code.

Information theoretic results in $[7,8]$ have shown that using multiple transmit and receive antennas improve the capacity of the system significantly in the presence of block fading channels. Different ST coded systems were proposed in [3,4,9-11] for quasi-static fading channels. Also, ST coded OFDM systems were considered in [12,13] and more recently in [14], where a low-density parity-check (LDPC) coded OFDM system with transmit diversity was presented. Many powerful ST codes have appeared in the literature as in [15-20]. However, trellis codes are suitable for low-complexity and short-delay applications due to their simple encoding and decoding algorithms.

If a trellis code is used on a block fading channel and the coded sequence is interleaved to break up the channel memory, then the effect of the channel memory is reduced significantly provided that the number of fading blocks is several times larger than the code constraint length [21]. Under this assumption the block fading channel can be approximated by a memoryless channel, and ST codes can be optimized accordingly with no significant loss in performance. Note that codes for singleantenna systems perform well under this assumption without the need for ST codes. However, consider wireless systems with multiple antennas at the base station. These antennas are used conventionally to provide receive diversity at the base station. However, the same antennas can be used for transmit diversity in the reverse link without imposing extra cost on the system. Motivated by this, this paper considers the design of good ST codes for rapid fading channels. Examples of QPSK and 8-PSK trellis codes optimized for rapid fading channels have appeared in [22,23]. However, the codes in [22] were based on computer search for the best codes and no systematic design approach is followed. This paper presents ST trellis codes employing QPSK and 16-QAM constellations using the set partitioning approach and optimized for rapid fading channels.

In this paper we propose the use of I-Q encoding technique to design better ST codes. Originally in [24], I-Q trellis codes for single-transmit antenna systems were shown to have high coding gains over conventional codes in rapid fading environments. This is due to the high minimum time diversity (MTD) provided by the I-Q encoding. Traditionally, increasing the MTD is achieved either by reducing the number of input bits to the encoder, which reduces the throughput, or increasing the memory of the encoder, which increases the complexity. By encoding the input stream using two parallel encoders, where each one encodes one dimension of the signal constellation, the MTD is increased with no penalty in the complexity. Hence, the I-Q approach is used to design more powerful trellis ST codes at the same delay and complexity as conventional ST codes using one encoder.

The paper is organized as follows. In Section II, the model of 


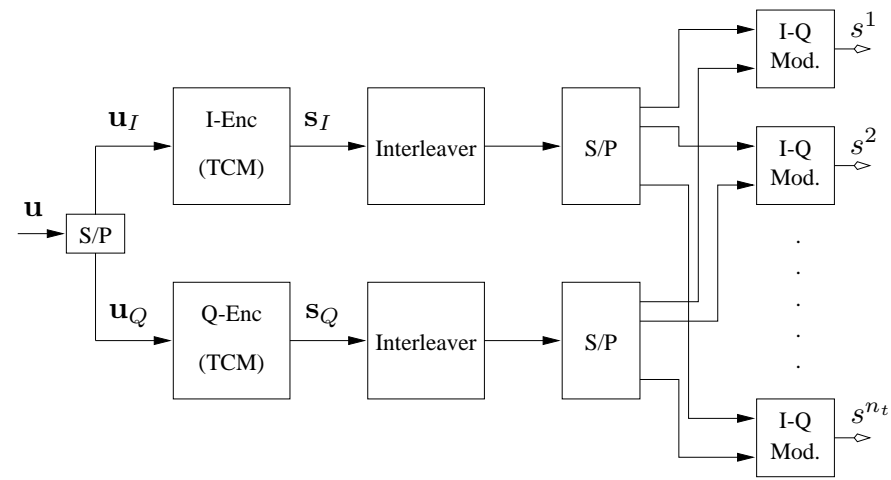

Fig. 1. The block diagram of the I-Q ST transmitter.

a trellis coded ST system is described. The proposed I-Q coded ST system and the decoding algorithm are presented in Section III. The new upper bounds on the PEP and BEP are derived in Section IV. Then, the ST codes based on the conventional and I-Q encoding schemes are presented in Section V. The performance of the proposed codes is compared with existing ST codes in Section VI. Finally, conclusions drawn from the work are discussed in Section VII.

\section{CONVENTIONAL ST SYSTEMS}

In this section, the model for trellis coded ST systems employing single encoders is described, which is similar to the system model in [4]. The transmitter consists of a trellis encoder, an interleaver, $n_{t}$ modulators and $n_{t}$ transmit antennas. During a frame transmission period of length $N T$, the input to the transmitter is a length- $N$ sequence $\mathbf{U}=\left\{\mathbf{u}_{l}\right\}_{l=1}^{N}$ of input vectors each of length $k$. Each component of the input vector $\mathbf{u}_{l}$ is assumed to be from a binary alphabet. The encoder produces a length- $N$ sequence $\mathbf{S}=\left\{\mathbf{s}_{l}\right\}_{l=1}^{N}$ of signal vectors each of length $n_{t}$. The $i^{t h}$ element of each signal vector $s_{l}^{i}$ is an element of an $M$-ary signal constellation, such as MPSK or $M$-QAM, which is modulated and transmitted using the $i^{\text {th }}$ transmit antenna in the time interval $l$. Therefore, the overall system throughput is $k / T$ bits/s. Before being modulated, the signal vectors are interleaved to avoid burst errors in the decoder.

The receiver consists of blocks that do the reverse processes of the corresponding blocks in the transmitter; namely, $n_{r}$ receive antennas, $n_{r}$ demodulators, a deinterleaver and a Viterbi decoder. The received signal at the $j^{\text {th }}$ receive antenna in time interval $l$ is

$$
y_{j, l}=\sqrt{E_{s}} \sum_{i=1}^{n_{t}} h_{j, l}^{i} s_{l}^{i}+z_{j, l}
$$

where $E_{s}$ is the average energy of the constellation used at each transmit antenna and $h_{j, l}^{i}$ is the channel gain between the $i^{t h}$ transmit and $j^{\text {th }}$ receive antennas at time interval $l$. The channel gains are modeled as independent zero-mean complex Gaussian random variables with unit variance, $\mathcal{C N}(0,1)$. Also, $z_{j, l}$ is an additive white noise modeled as $\mathcal{C N}\left(0, N_{0}\right)$. It is assumed that channels from different transmit antennas and channels to different receive antennas are uncorrelated. This is achieved by keeping the antennas apart by a distance greater than half the wave-

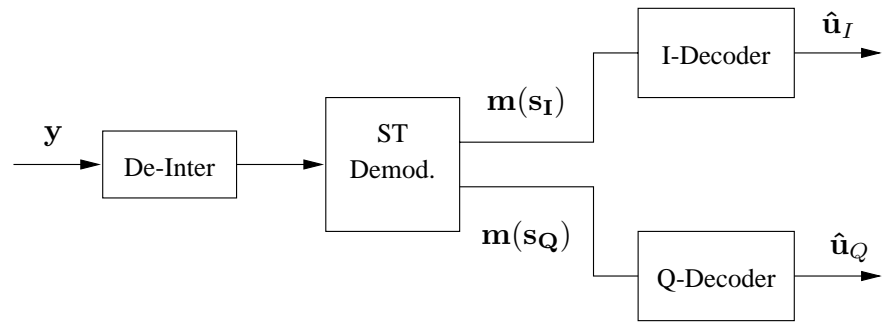

Fig. 2. The block diagram of the simplified decoding algorithm of I-Q ST codes.

length of the carrier [25]. The transmitted frame is decoded according to the maximum likelihood (ML) decoding rule, which is implemented using the Viterbi algorithm. In the following, the I-Q coded ST system is described.

\section{I-Q CODED ST SYSTEMS}

\section{A. Encoder}

The block diagram of the ST transmitter employing I-Q encoding is shown in Fig. 1. The input to the transmitter is as described in Section II. Each input vector $\mathbf{u}_{l}$ is split into two equal vectors $\mathbf{u}_{I, l}$ and $\mathbf{u}_{Q, l}$. The vector $\mathbf{u}_{I, l}$ is encoded by the I encoder into a signal vector $\mathbf{s}_{I, l}$ of length $n_{t}$, whose elements are drawn from an alphabet $\mathcal{A}_{\mathcal{I}}$ which is a 1-D constellation such as $M$-PAM. The 1 -D signals in $\mathbf{s}_{I, l}$ constitute the I components of the 2-D signals to be transmitted over the $n_{t}$ transmit antennas. The same applies to the $\mathrm{Q}$ branch, resulting in two length- $N$ sequences of 1-D signal vectors $\mathbf{S}_{I}=\left\{\mathbf{s}_{I, l}\right\}_{l=1}^{N}$ and $\mathbf{S}_{Q}=\left\{\mathbf{s}_{Q, l}\right\}_{l=1}^{N}$. After that, the I and $\mathrm{Q}$ codewords $\mathbf{S}_{I}$ and $\mathbf{S}_{Q}$ are interleaved. After interleaving, the 2-D signal $s_{l}^{i}$ to be transmitted over the $i^{\text {th }}$ antenna is drawn from the alphabet $\mathcal{A}=\mathcal{A}_{\mathcal{I}} \times \mathcal{A}_{\mathcal{Q}}$ according to: $s_{l}^{i}=s_{I, l}^{i}+j s_{Q, l}^{i}$, where $j=\sqrt{-1}$, resulting in a transmitted codeword $\mathbf{S}=\mathbf{S}_{I}+j \mathbf{S}_{Q}$.

\section{B. Decoder}

In this section, the subscript of the receive antenna is omitted to simplify notation. However, the discussion applies completely to multiple receive antennas. The received signal at each receive antenna (1) is expanded using complex representation as

$$
y_{l}=\sum_{i=1}^{n_{t}}\left(h_{I, l}^{i} s_{I, l}^{i}-h_{Q, l}^{i} s_{Q, l}^{i}\right)+j\left(h_{I, l}^{i} s_{Q, l}^{i}+h_{Q, l}^{i} s_{I, l}^{i}\right)+z_{l}
$$

where $h_{l}^{i}=h_{I, l}^{i}+j h_{Q, l}^{i}$. Define $\mathbf{Y}=\left\{y_{l}\right\}_{l=1}^{N}$ and $\mathbf{H}=$ $\left\{\mathbf{h}_{l}\right\}_{l=1}^{N}$, where $\mathbf{h}_{l}=\left\{h_{l}^{i}\right\}_{i=1}^{n_{t}}$ is a vector containing the fading gains from all transmit antennas at time $l$. The ML decoding rule at the $\mathrm{I}$ and $\mathrm{Q}$ decoders requires the computation of the likelihood functions $p\left(\mathbf{Y} \mid \mathbf{H}, \mathbf{S}_{I}\right)$ and $p\left(\mathbf{Y} \mid \mathbf{H}, \mathbf{S}_{Q}\right)$, respectively. However, due to the received mixture in (2), $\mathbf{Y}$ depends on both $\mathbf{S}_{I}$ and $\mathbf{S}_{Q}$. A straightforward solution is to use the super-trellis of the I and Q codes to compute the likelihood functions. In the super-trellis, the number of states is the product of the number of states of the I and Q codes, which prohibits its use for practical encoders' memory. For example, if each code had 32 states, the super-trellis would have $32 \times 32=1024$ states. Therefore, a low complexity decoder is of interest. 
A simplified decoding algorithm is proposed, which consists of a ST demodulator and two Viterbi decoders, one for each of the I and Q codes. The block diagram of this algorithm is shown in Fig. 2. In the ST demodulator, metrics corresponding to all possible pairs of signal vectors $\mathbf{s}=\left(\mathbf{s}_{I}, \mathbf{s}_{Q}\right)$ in the frame are computed using

$$
\mathbf{m}\left(y_{l}, \mathbf{s}\right)=\left|y_{l}-\sqrt{E_{s}} \sum_{i=1}^{n_{t}} h_{l}^{i} s^{i}\right|^{2} .
$$

When multiple receive antennas are used, the metric in (3) is modified to include maximal-ratio combining. Therefore, the following metrics are computed in the ST demodulator for time intervals $l=1 \ldots N$

$$
\left.\mathbf{m}\left(y_{l}, \mathbf{s}\right)\right|_{\mathbf{s}=\left(\mathbf{s}_{I}, \mathbf{s}_{Q}\right)}, \quad \forall \mathbf{s}_{I} \in \mathcal{A}_{\mathcal{I}}, \mathbf{s}_{\mathcal{Q}} \in \mathcal{A}_{\mathcal{Q}} .
$$

The metrics corresponding to the signal vectors $\mathbf{s}_{I}, \mathbf{s}_{Q}$ are computed as

$$
\begin{array}{cc}
\mathbf{m}_{l}\left(\mathbf{s}_{I}\right)=\left.\min _{\mathbf{s}_{Q} \in \mathcal{A}_{\mathcal{Q}}} \mathbf{m}\left(y_{l}, \mathbf{s}\right)\right|_{\mathbf{s}=\left(\mathbf{s}_{I}, \mathbf{s}_{Q}\right)}, & \mathbf{s}_{I} \in \mathcal{A}_{\mathcal{I}} \\
\mathbf{m}_{l}\left(\mathbf{s}_{Q}\right)=\left.\min _{\mathbf{s}_{I} \in \mathcal{A}_{\mathcal{I}}} \mathbf{m}\left(y_{l}, \mathbf{s}\right)\right|_{\mathbf{s}=\left(\mathbf{s}_{I}, \mathbf{s}_{Q}\right)}, & \mathbf{s}_{Q} \in \mathcal{A}_{\mathcal{Q}} .
\end{array}
$$

These metrics are fed to the I and Q Viterbi decoders, which apply separately the ML decoding rule for I and Q coded branches. Note that the complexity of this algorithm is linearly proportional to the number of states of the I and Q codes. However, the complexity of the ST demodulator is exponential in $n_{t}$. When a signal constellation of size $M$ is used at each transmit antenna, there are $M^{n_{t}}$ such metrics. In general, sphere decoding in $[26,27]$ can be used to reduce the complexity of the ST demodulator, especially for large number of transmit antennas. The performance of ST codes over rapid fading channels is presented in the following.

\section{PERFORMANCE ANALYSIS}

In this section, an upper bound on the PEP of ST codes over rapid fading channels is derived. The bound is tight and expressed in a product form, making it easy to use the TF of the codes to compute the BEP. The section starts with the derivation of the new bound and then the code TF is discussed and used to evaluate the BEP.

\section{A. Pairwise Error Probability (PEP)}

The subscripts $c, u$ and $b$ are used to denote conditional, unconditional and bit error probabilities, respectively. The conditional PEP is defined as the probability of decoding a received sequence as $\hat{\mathbf{S}}$ given that $\mathbf{S}$ was transmitted conditioned on the fading variables $\mathbf{H}=\left\{\mathbf{h}_{j, l}, j=1, \ldots, n_{r}, l=1, \ldots, N\right\}$. It is given by

$$
P_{c}(\mathbf{S}, \hat{\mathbf{S}})=\operatorname{Pr}(\mathbf{M}(\mathbf{Y}, \mathbf{S})-\mathbf{M}(\mathbf{Y}, \hat{\mathbf{S}})>0 \mid \mathbf{H}, \mathbf{S})
$$

where $\mathbf{M}(\mathbf{Y}, \mathbf{S})$ is the ML decoding metric corresponding to the codeword $\mathbf{S}$ given by

$$
\mathbf{M}(\mathbf{Y}, \mathbf{S})=\sum_{l=1}^{N} \sum_{j=1}^{n_{r}}\left|y_{j, l}-\sqrt{E_{s}} \sum_{i=1}^{n_{t}} h_{j, l}^{i} s_{l}^{i}\right|^{2} .
$$

Substituting (8) into (7) and going through some algebra, the conditional pairwise error probability simplifies to

$$
P_{c}=\frac{1}{2} \operatorname{erfc}\left(\sqrt{\frac{d_{E}^{2}(\mathbf{S}, \hat{\mathbf{S}})}{4 N_{0}}}\right)
$$

where

$$
d_{E}^{2}(\mathbf{S}, \hat{\mathbf{S}})=E_{s} \sum_{l=1}^{N} \sum_{j=1}^{n_{r}}\left|\sum_{i=1}^{n_{t}} h_{j, l}^{i}\left(s_{l}^{i}-\hat{s}_{l}^{i}\right)\right|^{2} .
$$

Using the matrix notation, the squared distance in (10) becomes

$$
d_{E}^{2}(\mathbf{S}, \hat{\mathbf{S}})=E_{s} \sum_{l=1}^{N} \sum_{j=1}^{n_{r}} \mathbf{h}_{j, l}^{*} A_{l} \mathbf{h}_{j, l}
$$

where (.)* denotes the complex conjugate of a complex vector. The matrix $A_{l}=\mathbf{e}_{l} \cdot \mathbf{e}_{l}^{*}$, where $\mathbf{e}_{l}=\left\{\left(s_{l}^{i}-\hat{s}_{l}^{i}\right)\right\}_{i=1}^{n_{t}}$ is a column vector. Then the conditional PEP is given by

$$
P_{c}=\frac{1}{2} \operatorname{erfc}\left(\sqrt{\frac{E_{s}}{4 N_{0}} \sum_{l=1}^{N} \sum_{j=1}^{n_{r}} \mathbf{h}_{j, l} A_{l} \mathbf{h}_{j, l}^{*}}\right) .
$$

Since $A_{l}$ is symmetric, its eigenvalue decomposition is written as: $A_{l}=V_{l}^{H} D_{l} V_{l}$, where the rows of $V_{l}$ are orthonormal and $D_{l}=\operatorname{diag}\left\{\lambda_{l}^{1}, \ldots \lambda_{l}^{\mathrm{n}_{\mathrm{t}}}\right\}$, with $\lambda_{l}^{i}$ is the $i^{\text {th }}$ eigenvalue of $A_{l}$. Here, $(.)^{H}$ denotes the Hermitian transpose of a matrix. Moreover, all $\lambda_{l}^{i}$ are zero except $\lambda_{l}=\left|\mathbf{e}_{l}\right|^{2}=\sum_{i=1}^{n_{t}}\left|e_{l}^{i}\right|^{2}$ because all the columns in $A_{l}$ are dependent on each other except one column. Let the vector $B_{j, l}=\mathbf{h}_{j, l} V_{l}^{H}$, and $d_{l}=\lambda_{l} \frac{E_{s}}{4 N_{0}}$, then the conditional PEP can be written as

$$
P_{c}=\frac{1}{2} \operatorname{erfc}\left(\sqrt{\sum_{l=1}^{N} d_{l} \sum_{j=1}^{n_{r}}\left|\beta_{j, l}\right|^{2}}\right) .
$$

Here the summation over the transmit antennas was removed since only one nonzero eigenvalue $\lambda_{l}$ exists, where $\beta_{j, l}$ is the element in $B_{j, l}$ corresponding to the nonzero eigenvalue. Since each $\beta_{j, l}$ is a normalized linear combination of Gaussian random variables, its distribution is $\mathcal{C N}(0,1)$, and hence $\left|\beta_{j, l}\right|^{2}$ is exponential random variable with parameter one. Define $\Gamma_{l}=\sum_{j=1}^{n_{r}}\left|\beta_{j, l}\right|^{2}$, then $\Gamma_{l}$ is an $n_{r}$-Erlang random variable with parameter one, whose probability density function (pdf) is

$$
f_{\Gamma}(\gamma)=\frac{1}{\left(n_{r}-1\right) !} \gamma^{\left(n_{r}-1\right)} e^{-\gamma}, \quad \gamma \geq 0 .
$$

Substituting $\Gamma_{l}$ in the expression of conditional PEP, it becomes

$$
P_{c}=\frac{1}{2} \operatorname{erfc}\left(\sqrt{\sum_{l=1}^{N} d_{l} \Gamma_{l}}\right) .
$$

The unconditional PEP is found by averaging (15) over the random variables $\left\{\Gamma_{l}\right\}_{l=1}^{N}$ and is written as

$P_{u}=\frac{1}{2} \int_{0}^{\infty} \cdots \int_{0}^{\infty} \operatorname{erfc}\left(\sqrt{\sum_{l=1}^{N} d_{l} \Gamma_{l}}\right)$ 


$$
\times f_{\Gamma}\left(\Gamma_{1}\right) \ldots f_{\Gamma}\left(\Gamma_{N}\right) d \Gamma_{1} \ldots d \Gamma_{N} .
$$

Doing the following change of variables: $\delta_{l}=\frac{d_{l}}{1+d_{l}}$ and $\omega_{l}=$ $\Gamma_{l}\left(1+d_{l}\right)$ and using pdf transformation, the PEP becomes

$$
\begin{aligned}
P_{u} & =\frac{1}{2} \prod_{l \in \eta} \frac{1}{\left(1+d_{l}\right)^{n_{r}}} \int_{0}^{\infty} \ldots \int_{0}^{\infty} \operatorname{erfc}\left(\sqrt{\sum_{l=1}^{N} \delta_{l} \omega_{l}}\right) \\
& \times \exp \left(\sum_{l=1}^{N} \delta_{l} \omega_{l}\right) f_{\omega}\left(\omega_{1}\right) \ldots f_{\omega}\left(\omega_{|\eta|}\right) d \omega_{1} \ldots d \omega_{|\eta|},
\end{aligned}
$$

where $\eta=\left\{l: \mathbf{e}_{l} \neq 0\right\}$ and $|\eta|$ is the size of $\eta$. Define $L=\min |\eta|$ (the MTD of the ST code) and $\delta_{\min }=$ $\min \left\{\delta_{l}\right\}_{l=1}^{|\eta|}$. Note that $\sum_{l=1}^{N} \delta_{l} \omega_{l} \geq \delta_{\min } \sum_{l=1}^{N} \omega_{l}$ and the function $\operatorname{erfc}(\mathrm{x}) \exp (\mathrm{x})$ is monotonically decreasing, then the PEP is upper bounded as

$$
\begin{aligned}
P_{u} \leq \frac{1}{2} \prod_{l=1}^{L} \frac{1}{\left(1+d_{l}\right)^{n_{r}}} \int_{0}^{\infty} & \operatorname{erfc}\left(\sqrt{\delta_{\min } \Omega}\right) \\
& \times e^{\left(\delta_{\min } \Omega\right)} f_{\Omega}(\Omega) d \Omega,
\end{aligned}
$$

where $\Omega=\sum_{l=1}^{N} \omega_{l}$, which is a sum of $L$ independent $n_{r^{-}}$ Erlang random variables, has an $n_{r} L$-Erlang distribution with parameter one. The pdf of $\Omega$ is given by (14) with replacing $n_{r}$ by $n_{r} L$. Substituting this pdf, the PEP becomes

$$
\begin{gathered}
P_{u \leq} \frac{1}{2\left(n_{r} L-1\right) !} \prod_{l=1}^{L} \frac{1}{\left(1+d_{l}\right)^{n_{r}}} \int_{0}^{\infty} \operatorname{erfc}\left(\sqrt{\delta_{\min } \Omega}\right) \\
\times e^{\left(\delta_{\min } \Omega\right)} \Omega^{\left(n_{r} L-1\right)} d \Omega .
\end{gathered}
$$

This integral is evaluated using an equality in [28] and the resultant expression for the PEP is

$$
\begin{aligned}
P_{u} \leq \frac{1}{2\left(n_{r} L-1\right) !} & \sum_{j=1}^{n_{r} L}\left(\begin{array}{c}
2 n_{r} L-j-1 \\
n_{R} L-1
\end{array}\right)\left(\frac{2}{1+\sqrt{\delta_{\min }}}\right)^{j} \\
& \times \prod_{l=1}^{L} \frac{1}{\left(1+d_{l}\right)^{n_{r}}} .
\end{aligned}
$$

By employing the union bound and averaging over all transmitted codewords we obtain an upper bound on the error event probability $P_{e, u}$

$$
P_{e, u} \leq \sum_{\mathbf{S}} \sum_{\hat{\mathbf{S}}} P(\mathbf{S}) P_{u}(\mathbf{S}, \hat{\mathbf{S}}),
$$

where $P(\mathbf{S})$ is the probability of a codeword $\mathbf{S}$. If the trellis code satisfies Zehavi and Wolf criteria [29], the error event probability in (21) can be computed by assuming that the all-zero codeword $\mathbf{S}_{\mathbf{0}}$ was sent and

$$
P_{e, u} \leq \sum_{\hat{\mathbf{S}}} P_{u}\left(\mathbf{S}_{\mathbf{0}}, \hat{\mathbf{S}}\right)
$$

In order to evaluate (22) and to find the corresponding BEP, we need to enumerate the number of codewords with certain Euclidean distance from the all-zero codeword. This is accomplished using the TF of the ST code as described below.

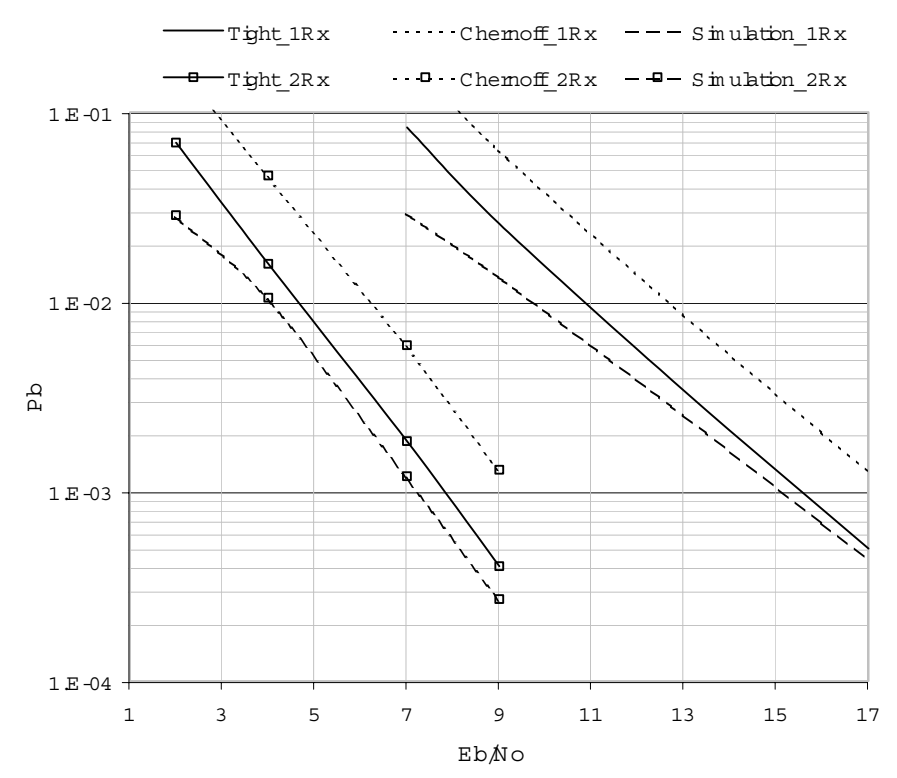

Fig. 3. Bounds and simulation of the 4-state QPSK code in [4].

\section{B. Transfer Function (TF)}

In this subsection, we describe how to evaluate the TF of a ST code. The transfer function of a trellis code enumerates the number of codewords at every input weight and output distance and can be calculated using the error state diagram [30]. The label associated with each branch connecting two states in the error state diagram depends on the error vector between the signal associated with that branch and the zero signal vector $\mathbf{s}_{0}$, i.e., $\mathbf{e}=\mathbf{s}-\mathbf{s}_{0}$. If an $M$-ary signal constellation is used at each transmit antenna, there are $M^{n_{t}}-1$ different error vectors. Denote the distinct squared Euclidean distances from $\mathbf{s}_{0}$ as $\left\{\xi_{1}, \xi_{2}, \ldots, \xi_{m}\right\}=\left\{\xi: \xi=E_{s}\left\|\mathbf{s}-\mathbf{s}_{0}\right\|^{2}, \mathbf{s} \in \mathcal{A}^{n_{t}}\right\}$, where $\|\mathbf{x}\|^{2}$ denotes the norm of a vector $\mathbf{x}$. In the error state diagram, each branch is labeled by $J^{u} D_{1}^{v_{1}} \ldots D_{m}^{v_{m}}$ where $v_{l}=1$ if the corresponding signal vector has distance $\xi_{l}$. Also, the exponent of the variable $J$ is the weight of the input vector causing the transition. For example, a state transition with input weight $u$ and signal vector with distance $\xi_{l}$ is represented by $J^{u} D_{l}$. The reader is referred to [30] for the complete details of computing the transfer function of trellis codes. The transfer function of the ST code is written as

$$
\begin{array}{r}
T\left(J, D_{1}, \ldots, D_{m}\right)=\sum_{u} \sum_{v_{1}, \ldots, v_{m}} a\left(u, v_{1}, \ldots, v_{m}\right) \\
\times J^{u} D_{1}^{v_{1}} \ldots D_{m}^{v_{m}},
\end{array}
$$

where $a\left(u, v_{1}, \ldots, v_{m}\right)$ is the number of codewords with input weight $u$ and $v_{i}$ error vectors with distance $\xi_{i}$ from $\mathbf{s}_{0}$, for $i=$ $1, \ldots, m$.

\section{Bit Error Probability (BEP)}

Using the new bound in (20) and comparing the expressions in (22) and (23), then the error event probability and the BEP are written, respectively as

$$
P_{e, u} \leq \frac{1}{2^{2 n_{r} L}} \sum_{j=1}^{n_{r} L}\left(\begin{array}{c}
2 n_{r} L-j-1 \\
n_{r} L-1
\end{array}\right)\left(\frac{2}{1+\sqrt{\delta_{\min }}}\right)^{j}
$$




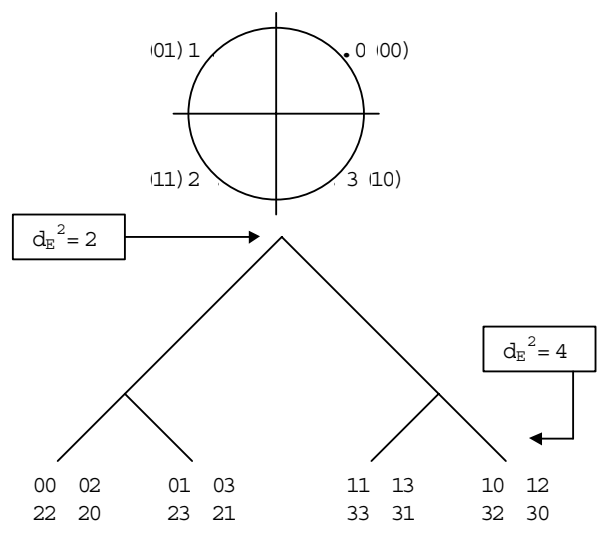

Fig. 4. The 4-D QPSK signal space partitioning.

$$
\begin{gathered}
\times\left. T\left(J, D_{1}, \ldots, D_{m}\right)\right|_{J=1, D_{v}=\left(1+\frac{\xi_{v}}{4 N_{0}}\right)^{-n_{r}}, v=1, \ldots, m} \\
P_{b} \leq \frac{1}{k}\left[\frac{1}{2^{2 n_{r} L}} \sum_{j=1}^{n_{r} L}\left(\begin{array}{c}
2 n_{r} L-j-1 \\
n_{r} L-1
\end{array}\right)\left(\frac{2}{1+\sqrt{\delta_{\min }}}\right)^{j}\right] \\
\times\left.\frac{\partial T\left(J, D_{1}, \ldots, D_{m}\right)}{\partial J}\right|_{J=1, D_{v}=\left(1+\frac{\xi_{v}}{4 N_{0}}\right)^{-n_{r}}, v=1, \ldots, m}, \quad
\end{gathered}
$$

where $\delta_{\min }=\min \left\{\frac{\xi_{l} / 4 N_{0}}{1+\xi_{l} / 4 N_{0}}, l=1, \ldots, m\right\}$ and $k$ is the number of input bits to the encoder. The corresponding Chernoff bound in [4] as a function of the TF is written as

$$
P_{b} \leq\left.\frac{1}{k} \frac{\partial T\left(J, D_{1}, \ldots, D_{m}\right)}{\partial J}\right|_{J=1, D_{v}=\left(1+\frac{\xi_{v}}{4 N_{0}}\right)^{-n_{r}}, v=1, \ldots, m .}
$$

The bounds in (25) and (26) were evaluated for the 4-state QPSK ST code in [4]. The curves corresponding to the bounds and simulation are shown in Fig. 3. It is observed that the Chernoff bound is loose where the new bound is tight to the simulation curve. The designed codes are presented in the following.

\section{THE PROPOSED ST CODES}

In this section we describe the proposed ST codes employing single encoders and the I-Q encoding scheme. The design criteria of ST codes over rapid fading channels were derived in [4] and appear in (20). The design criteria are summarized in maximizing:

1. The MTD of the code, i.e., $L$.

2. The minimum squared product distance defined as: $d_{p}^{2}=$ $E_{s} \prod_{l=1}^{L} \sum_{i=1}^{n_{t}}\left|s_{l}^{i}-\hat{s}_{l}^{i}\right|^{2}$.

These two criteria are used below to design trellis ST codes that use either a single encoder or the I-Q technique. The codes are designed using the set partitioning approach and use QPSK and 16-QAM constellations and two transmit antennas i.e., $n_{t}=2$. From the second rule, the set partitioning is performed in the same way as in additive white Gaussian noise (AWGN) channels. In this approach, the distance $d_{E}^{2}=E_{s} \sum_{i=1}^{n_{t}}\left|s^{i}-\hat{s}^{i}\right|^{2}$ between signal vectors at branches diverging from or remerging

$$
\begin{aligned}
& 00,02,22,20 \\
& 21,23,03,01 \\
& 33,31,11,13 \\
& 12,10,30,32
\end{aligned}
$$

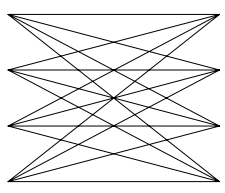

Fig. 5. Trellis diagram of the 4-state QPSK2 code.

to the same state is maximized. To maximize $d_{E}^{2}$, the $2 n_{t}$-D signal space is partitioned into subsets such that $d_{E}^{2}$ between signal vectors in the same subset is increased. If $d_{E}^{2}$ cannot be increased anymore, partitioning is performed so that $d_{P}^{2}$ between signal vectors in the same subset is increased. As a result, both $d_{E}^{2}$ and $d_{P}^{2}$ between pairs in the same subset are maximized. The detailed description of the codes are discussed in the following.

\section{A. Single-Encoder Codes}

All QPSK codes presented in this paper have a throughput of $k=2$ bits/s, i.e., the encoder receives two bits every time interval and outputs two QPSK signals. The first proposed code is referred to as QPSK2. The set partitioning of a 4-dimensional QPSK signal space is shown in Fig. 4. In the code trellis, signal vectors labeling branches leaving the same state are taken from the same subset, and hence, signal vectors of these branches have the maximum possible $d_{E}^{2}$ and $d_{P}^{2}$. The trellis diagram of the 4-state code is shown in Fig. 5. From the trellis, the MTD of the code is 2 and $d_{P}^{2}=24$ with corresponding error event [0 2;2 1]. Note that the performance of this code is equivalent to that of the code in [22] since they have the same design parameters.

The QAM codes presented in this paper have a throughput of $k=4$ bits/s. Therefore, the encoder receives 4 bits every time interval and outputs two signal points from a Gray-mapped 16-QAM constellation. The first proposed scheme is referred to as QAM2. Partitioning the 4-D 16-QAM signal space in a systematic way followed in the QPSK case is difficult. Hence, a simple approach that produces good $d_{P}^{2}$ is proposed, which is a modified version of the permutation method in [31]. In this method, the allowed 16-QAM signal vectors labeling branches leaving or emerging into the same state are forced to be different in both symbols. The method starts with listing all the possible 16-QAM symbols in order, starting by $s_{0}$ and ending with $s_{15}$ in a $16 \times 1$ vector. Then a $16 \times 2$ vector is formed by listing all the pairs of same first and second symbols and denoted by $A_{00}$. Denote the vector that has the second column of $A_{00}$ shifted by $i$ rows by $A_{i 0}$. Similarly, when the vector $A_{i 0}$ is shifted by $j$ rows it is denoted by $A_{i j}$. The labels of branches leaving each state are taken as the rows of the vectors having the maximum Hamming distance from each other. The trellis diagram of the 16-state QAM2 code is shown in Fig. 6. This code has a MTD equal to 2 and $d_{P}^{2}=3.2$ with the corresponding error event is [1 $1 ; 157]$. The I-Q based codes are considered in the following.

\section{B. I-Q Codes}

The I-Q QPSK codes use BPSK constellation as the 1-D constellation at the output of the I and Q encoders. The set partition- 

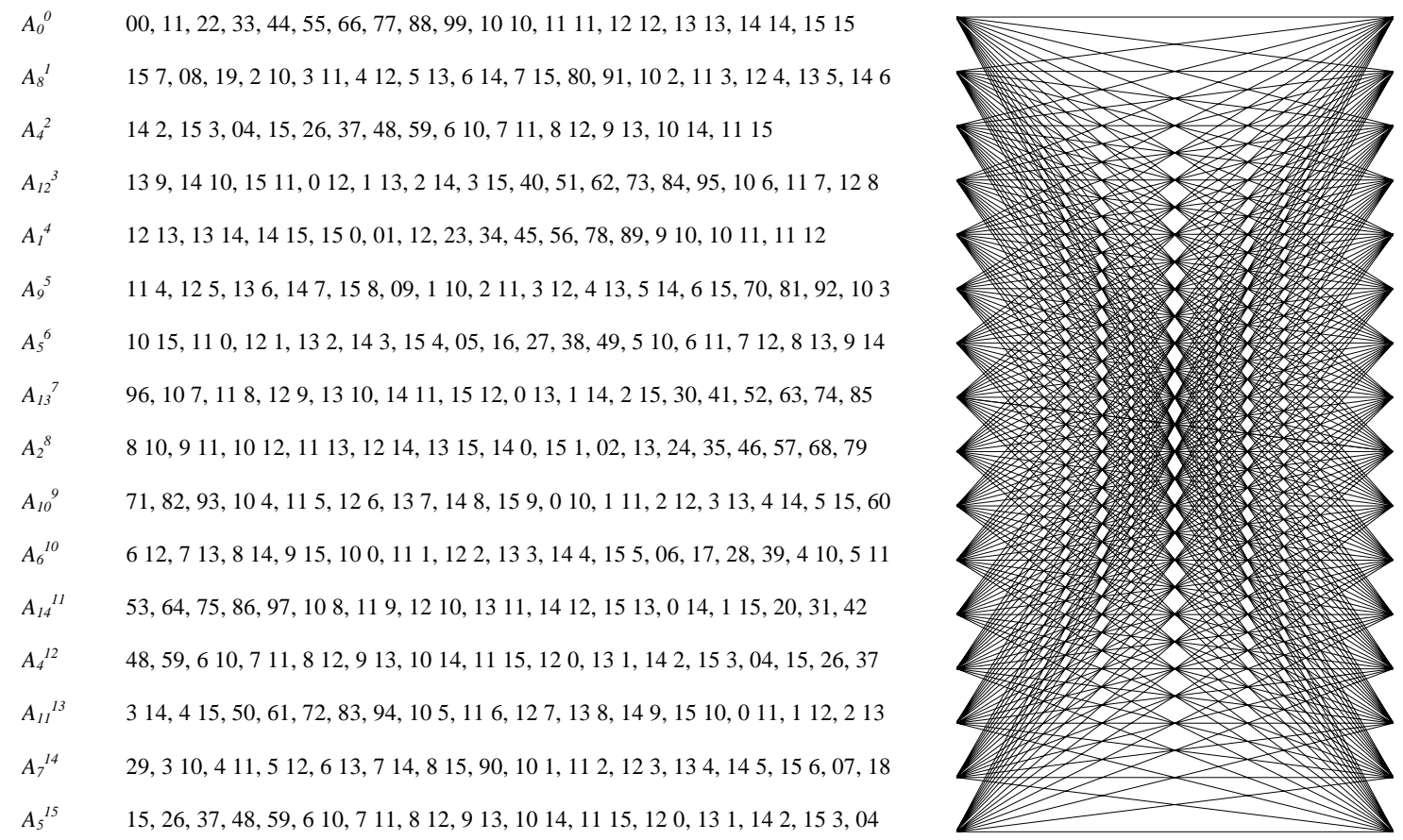

Fig. 6. Trellis diagram of the 16-state QAM2 code.

ing described above is followed for the 2-D BPSK signal space. This is straightforward since only four signals exist in the space. The trellis diagrams of the 4-state and 8-state codes are shown in Fig. 7. Note the labels of branches leaving or remerging to the same state differ in both symbols, which is similar to designing codes for single-antenna systems over fading channels. The resultant MTD values are 3 and 4 for the 4 -state and 8 -state codes, respectively. The increase in the MTD values resulting from using the I-Q encoding scheme will be clearly observed in the code performance. The product distance of the I-Q ST codes cannot be calculated unless the super-trellis is formed. For comparison purposes, it is computed in one dimension only. For example, the 4-state I-Q code has an MTD of 3 and $d_{P}^{2}=5.66$ in one dimension with corresponding error event $[11 ; 10 ; 11]$.

The I-Q 16-QAM ST code uses 4-AM constellation at the output of the I and Q encoders. The signal space to be partitioned is the 2-D 4-AM signal space, which is shown in Fig. 8. As in above codes, partitioning is performed such that $d_{E}^{2}$ and $d_{P}^{2}$ of the generated subsets are higher each time the partitioning is performed. The trellis diagrams of the 4 and 32-state codes are shown in Fig. 9 and 10, respectively. From the figures, the labels of branches departing or remerging to the same state are chosen from different subsets in the last partitioning level. This maximizes the MTD of the 4, and 32-state codes to 2 and 4, respectively. For the 4 -state code, $d_{P}^{2}=3.2$ in one dimension with corresponding error event [0 3;23], where $d_{P}^{2}=7.2$ for the 32 -state with corresponding error event [0 3;2 3;3 0;3 1]. The difference in the MTD compared to conventional trellis codes is a result of using the I-Q encoding scheme.

In general, the complexity of a trellis code is equal to the total number of branches leaving all states divided by the associated
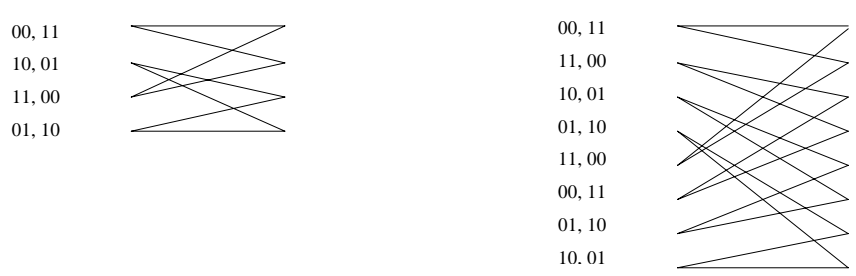

Fig. 7. Trellis diagrams of the I-Q QPSK codes (4 and 8-state).

information bits with each transition [24]. The complexity of the I-Q QPSK codes, with the simplified decoding algorithm, is the same as the QPSK2 code. However, the complexity of the 32state I-Q code, decoded by the simplified algorithm, is 64 , which is the same as that of the 16-state QAM2 code. If the supertrellis decoding is used, the 4-state I-Q code has a complexity of 64. Therefore, for fair comparison, the 16-state QAM2 code is compared with the 32-state I-Q code with the simplified decoding, and to the 4-state I-Q code with the super-trellis decoding.

\section{RESULTS}

This section presents simulation results of the new codes over three fading channels. The first one is perfectly interleaved channel, which is equivalent to a rapid fading channel. The other two channels have fading rates $f_{D} T$ of 0.01 and 0.005 and interleaved using a $25 \times 16$ block interleaver, where $f_{D}$ is the Doppler spread of the channel. An interleaver is considered to be sufficient if it has a depth on the order of $1 / 4 f_{D} T$ and a span larger than the code constraint length. Note that the interleave 


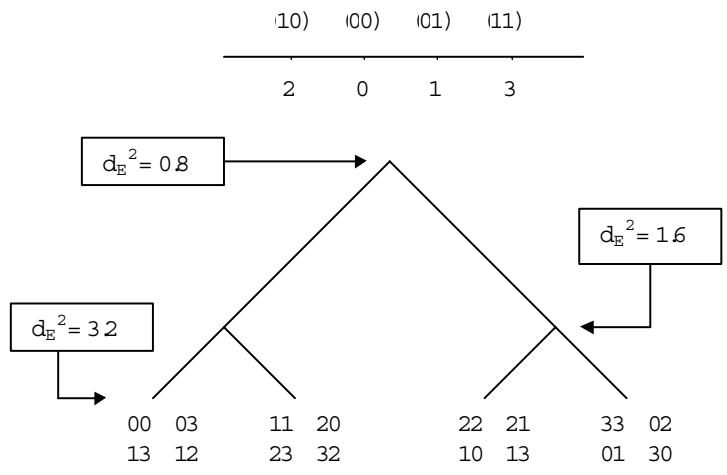

Fig. 8. 2-D 4-AM signal space partitioning.

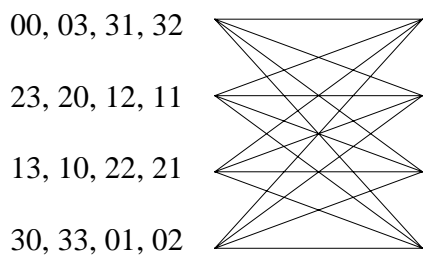

Fig. 9. Trellis diagram of the 4-state I-Q 16-QAM code.

$00,03,31,32$

$23,20,12,11$

$13,10,22,21$

$30,33,01,02$

$03,31,32,00$

$20,12,11,23$

$10,22,21,13$

$33,01,02,30$

$03,00,32,31$

$20,23,11,12$

$10,13,21,22$

$33,30,02,01$

$31,03,00,32$

$12,20,23,11$

$22,10,13,21$

01, 33, 30, 02

$31,32,00,03$

$12,11,23,20$

$22,21,13,10$

$01,02,30,33$

$32,00,03,31$

$11,23,20,12$

$21,13,10,22$

$02,30,33,01$

$32,03,31,00$

$11,12,20,23$

$21,22,10,13$

$02,01,33,30$

$00,32,31,03$

$23,11,12,20$

$13,21,22,10$

$30,02,01,33$

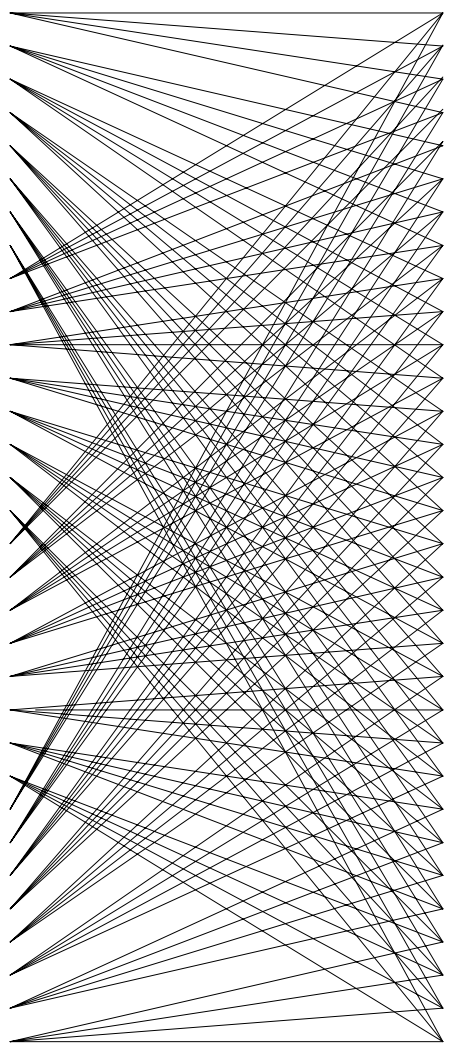

Fig. 10. Trellis diagram of the 32-state I-Q 16-QAM code.

is sufficient for the channel with $f_{D} T=0.01$ but insufficient for the channel with $f_{D} T=0.005$. Since the new codes are optimized for rapid fading channels, it is important to test their robustness under imperfect conditions; namely, a sufficiently interleaved and an insufficiently interleaved channels.
Table 1. The parameters of the designed codes.

\begin{tabular}{|l|c|c|}
\hline The Code & MTD & $d_{P}^{2}$ \\
\hline \hline QPSK1 & 2 & 4 \\
\hline QPSK2 & 2 & 24 \\
\hline I-Q QPSK (4-s) & 3 & 5.66 \\
\hline QAM1 & 2 & 0.64 \\
\hline QAM2 & 2 & 3.2 \\
\hline I-Q QAM (4-s) & 2 & 3.2 \\
\hline I-Q QAM (32-s) & 4 & 7.2 \\
\hline
\end{tabular}

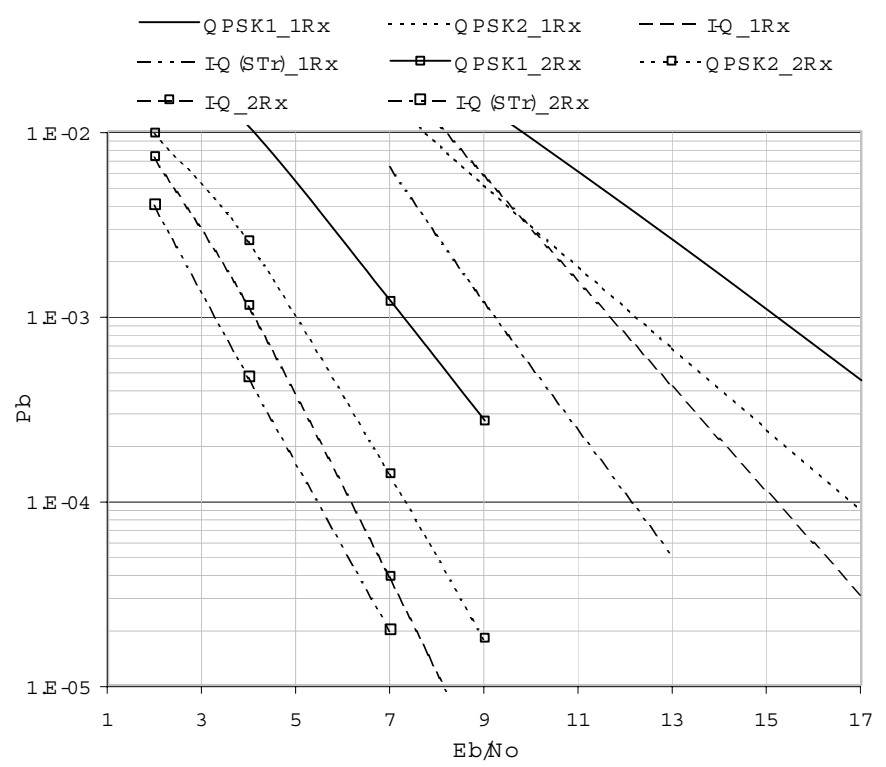

Fig. 11. Performance of the 4-state QPSK codes over a rapid fading channel (Rx: receive antenna, STr: Super Trellis).

\section{A. QPSK Codes}

The ST QPSK code, referred to as QPSK1, is presented here as a baseline for comparison with the proposed codes. The MTD of the 4-state code is 2 and $d_{P}^{2}=4$. The new codes (QPSK2 and I-Q) are compared with the QPSK1 code in Fig. 11 over a rapid fading channel. The QPSK2 code provides $2 \mathrm{~dB}$ gain over the QPSK1 code because it has higher $d_{P}^{2}$ value. Moreover, the I-Q code with super-trellis decoding provides $3 \mathrm{~dB}$ over the QPSK1 at bit error rate (BER) of $10^{-3}$. However, the decoding complexity of the super-trellis decoding of this code has increased from 8 to 32 . The I-Q code, using the simplified decoding algorithm provides a coding gain of $3 \mathrm{~dB}$ over QPSK1 and more than $0.5 \mathrm{~dB}$ over QPSK2 at BER of $10^{-3}$. Although the product distance of the I-Q code is lower than that of the QPSK2, its MTD is higher, which is more important. Note that the gains of the I-Q codes increase as the high signal-to-noise ratio (SNR) increase, where the simplified decoding algorithm works well. Also, the slope of its performance curve versus the SNR is larger than those of the QPSK1 and QPSK2 codes. This is expected since the MTD of the I-Q code is higher, which controls the slope of the curve.

The same trend is observed when the receiver has two receive antennas. Generally, the coding gain is expected to decrease because using two receive antennas provides a form of diversity 


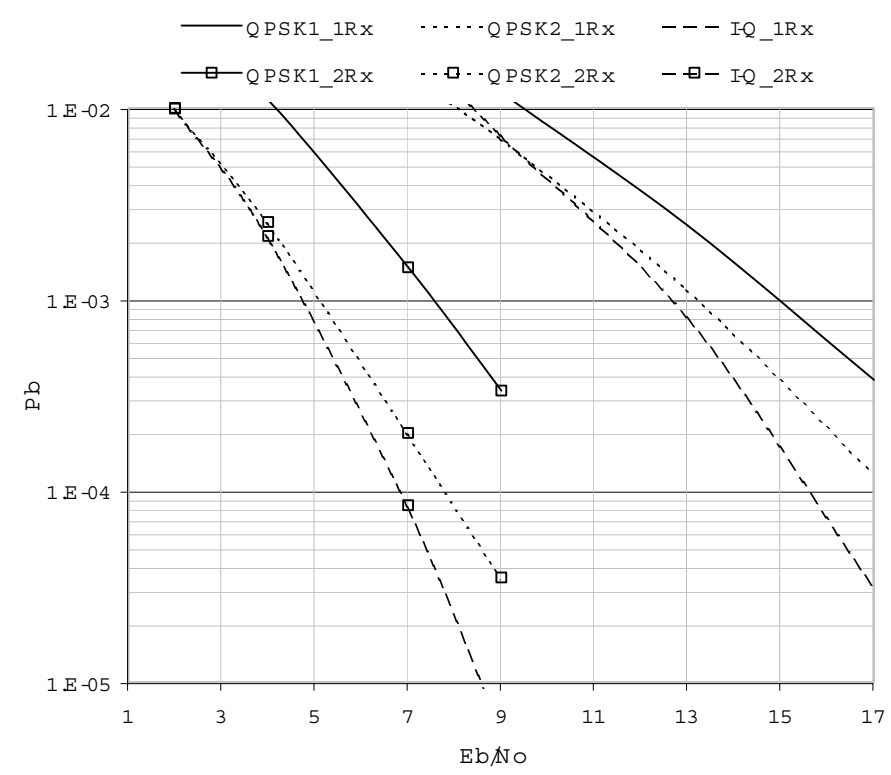

Fig. 12. Performance of the 4-state QPSK codes over a sufficiently interleaved fading channel with $f_{D T}=0.01$ (Rx: receive antenna).

and hence, the time diversity provided by the code becomes less important. However, the results show that the gain of the I-Q code, using the simplified decoding algorithm, over the other two codes increases compared to the one-receive antenna case. The reason behind this is that the two receive branches add a form of diversity in the ST demodulator. This reduces the suboptimality of the simplified decoding algorithm. The gains of the I-Q code over QPSK1 and QPSK2 codes are more than $3 \mathrm{~dB}$ and $1 \mathrm{~dB}$, respectively. Hence, the simplified algorithm provides better performance for the case of two receive antennas.

Fig. 12 shows the performance of the 4-state codes over a sufficiently interleaved channel with $f_{D} T=0.01$. It is obvious that the I-Q coding scheme is still the best followed by QPSK2. The gains of the first two codes over the last one are slightly reduced due to the non-ideal interleaving. However, the gains reduction is small showing the robustness of the codes under imperfect conditions. The case of insufficient interleaved fading channel with $f_{D} T=0.005$ is shown in Fig. 13. The same trends are observed but with less relative gains. It is worth mentioning that the designed codes are slightly sensitive to slow environments. In [21], a small performance degradation was observed when the I-Q ST codes were tested over block fading channels.

\section{B. 16-QAM Codes}

The 16-QAM ST code, referred to as QAM1, was optimized in [4] for quasi-static fading channels. It is presented as a baseline for comparison with the proposed codes. The MTD of this code is 2 and its $d_{P}^{2}=0.64$. The performance of QAM1 and QAM2 codes over an ideally interleaved fading channel is shown in Fig. 14. The figure also shows the performance of the 4-state and the 32-state I-Q code. The 4-state code is decoded using super-trellis decoding and the 32-state is decoded using the simplified decoding algorithm, resulting in the same complexity as QAM1 and QAM2 codes. In the case of one re-

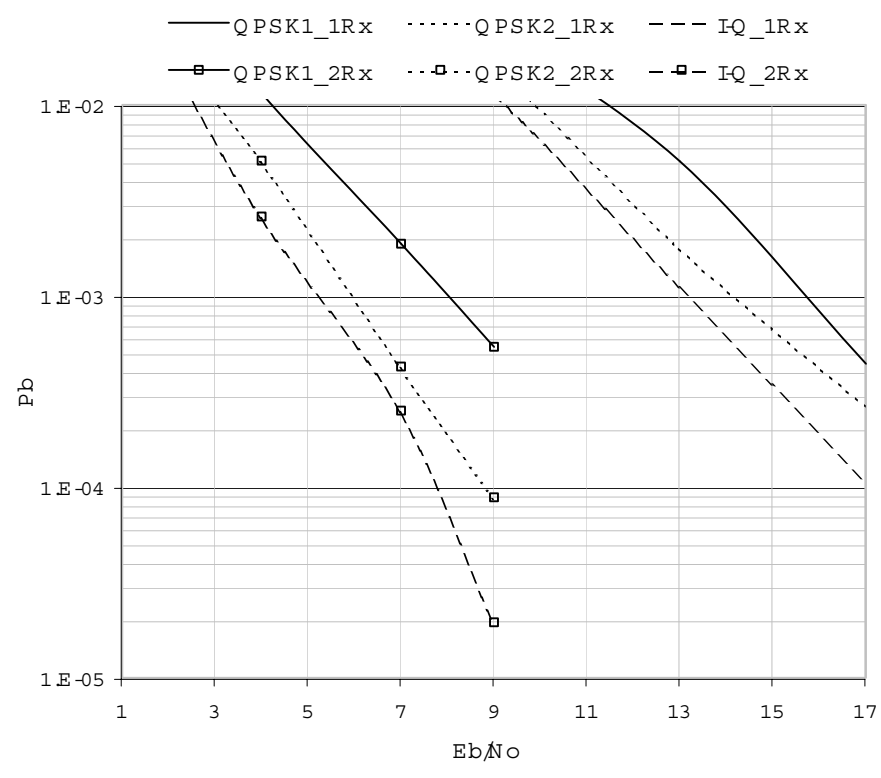

Fig. 13. Performance of the 4-state QPSK codes over an insufficiently interleaved fading channel with $f_{D T}=0.005$ (Rx: receive antenna).

ceive antenna, the gains of the 4-state I-Q code over QAM1 and QAM2 codes are 2.5 and $0.5 \mathrm{~dB}$, respectively. In the two receive antennas case, the above gains are increased to 2 and $0.3 \mathrm{~dB}$, respectively. On the other hand, gains of the 32-state I-Q code over QAM1, QAM2 and the 4-state code with super-trellis decoding are $2.5,1$ and $0.5 \mathrm{~dB}$, respectively. Although QAM2 and the 4-state I-Q codes have the same MTD and product distances, the I-Q codes outperform QAM2. This is due to the fact that the product distance is computed in one dimension and hence the effective product distance is expected to be higher. The same codes are tested over the sufficiently interleaved channel with $f_{D} T=0.01$ and the results are shown in Fig. 15. Similar to the QPSK codes, the gains are slightly reduced, showing the robustness of the new codes.

Note that the performance of the simplified decoding algorithm is significantly degraded in the case of single-receive antenna, and good in the two-receive antenna case. This is due to the diversity used by the ST demodulator in the two-receive antenna case. Also, the simplified algorithm performs worse than the QPSK code for the single-receive antenna case. We conclude from this that the algorithm degrades the performance for nonconstant energy constellations, and its performance is acceptable for constant-energy constellations. The last observation is that the degradation of the performance of the simplified algorithm decreases as the SNR is increased. This is due to the sensitivity of the ST demodulator to the received SNR, where its metrics reliability increases as the SNR is increased.

\section{CONCLUSIONS}

New trellis ST codes based on the QPSK and 16-QAM signal constellations are proposed for rapid fading channels. Two encoding schemes were employed in designing the codes. The first scheme uses single encoder whereas the second scheme uses the I-Q encoding technique. The design process exploits the set par- 


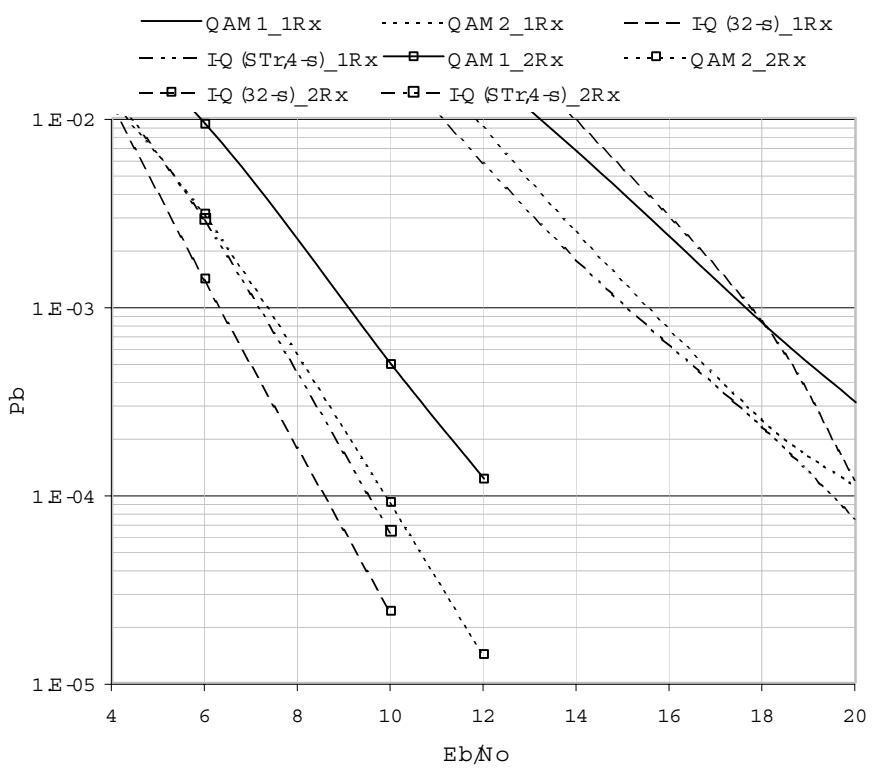

Fig. 14. Performance of the 16-QAM codes over a rapid fading channel (Rx: receive antenna, STr: Super Trellis).

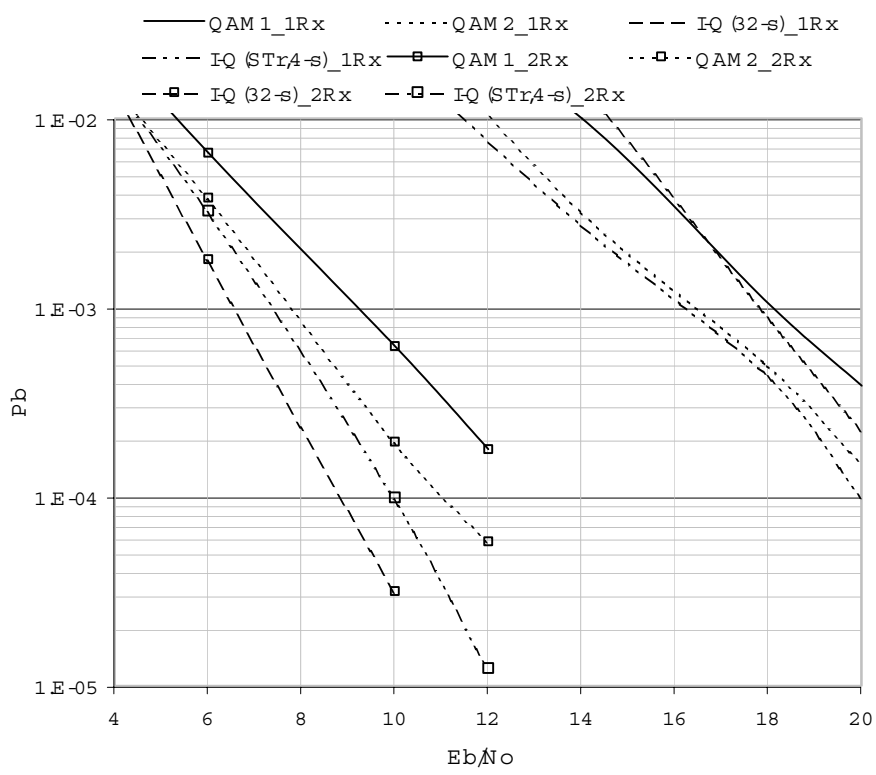

Fig. 15. Performance of the 16-QAM codes over a sufficiently interleaved fading channel with $f_{D T}=0.01(\mathrm{Rx}$ : receive antenna, STr: Super Trellis).

titioning approach to maximize the design parameters. Results show that these codes are robust in different interleaving environments. A simplified decoding algorithm for I-Q ST codes is proposed and used instead of the super-trellis decoding. It was shown that this algorithm performs near optimum in the cases of two-receive antennas or constant-energy signal constellations.

\section{ACKNOWLEDGEMENTS}

The authors would like to acknowledge the support of King Fahd University of Petroleum and Minerals provided to conduct this research.

\section{REFERENCES}

[1] R. J. McEliece and W. E. Stark, "Channels with block iinterference," IEEE Trans. Inform. Theory, vol. 30, pp. 44-53, Jan. 1984.

[2] E. Biglieri, J. Proakis, and S. Shamai, "Fading channels: informationtheoretic and communications Aspects," IEEE Trans. Inform. Theory, vol. 44, pp. 2619-2692, Oct. 1998.

[3] G. Foschini, "Layered space-time architecture for wireless communication in a fading environment when using multi-element antennas," Bell Labs. Technical Journal, vol. 1, no. 2, pp. 41-59, 1996.

[4] V. Tarokh, N. Seshadri, and A. Calderbank, "Space-time codes for high data rate wireless communication: performance criterion and code construction," IEEE Trans. Inform. Theory, vol. 44, pp. 744-765, Mar. 1998.

[5] S. Alamouti, "A simple transmit diversity technique for wireless communications," IEEE J. Select. Areas in Commun., vol. 16, pp. 1451-1458, Oct. 1998.

[6] M. Uysal and C. Georghiades, "Error performance analysis of space-time codes over rayleigh fading channels," J. Commun. Networks(JNC), vol. 2, pp. 351-356, Dec. 2000.

[7] E. Telatar, "Capacity of multi-antenna gaussian channels," AT\&T Bell Laboratories Internal Tech. Memo., 1995.

[8] T. Marzetta and B. Hochwald, "Capacity of a mobile multiple-antenna communication link in rayleigh flat fading," IEEE Trans. Inform. Theory, vol. 45, pp. 139-157, Jan. 1999.

[9] V. Tarokh, H. Jafarkhani, and A. Calderbank, "Space-time block codes from orthogonal designs," IEEE Trans. Informa. Theory, vol. 45, pp. 1456-1467, Jul. 1999.

[10] B. Hochwald and T. Marzetta, "Unitary space-time modulation for multiple-antenna communications in rayleigh flat fading," IEEE Trans. Inform. Theory, vol. 46, pp. 543-564, Mar. 2000.

[11] B. Hochwald and W. Sweldens, "Differential unitary space-time modulation," IEEE Trans. Commun., vol. 48, pp. 2041-2052, Dec. 2000.

[12] D. Agrawal et al. "Space-time coded OFDM for high data-rate wireless communication over wideband channels," in Proc. VTC'98, May 1998, pp. 2232-2236.

[13] R. Blum et al. "Improved space-time coding for MIMO-OFDM wireless communications," IEEE Trans. Commun., vol. 49, pp. 1873-1878, Jan. 2001.

[14] B. Lu, X. Wang, and K. Narayanan, "LDPC-based space-time coded OFDM systems over correlated fading channels: performance analysis and receiver design," IEEE Trans. Commun., vol. 50, pp. 74-88, Jan. 2002.

[15] A. Naguib et al. "A space-time coding modem for high-data-rate wireless communications," IEEE J. Select. Areas Commun., vol. 16, pp. 14591478, Oct. 1998.

[16] X. Lin and R. Blum, "Improved space-time codes using serial concatenation," IEEE Commun. Lett., vol. 4, pp. 221-223, Jul. 2000.

[17] Y. Liu, M. Fitz, and O. Takeshita, "Full Rate Space-Time Turbo Codes," IEEE J. Select. Areas Commun., vol. 19, pp. 969-980, May 2001.

[18] D. Cui and A. Haimovich, "Design and performance of turbo space-time coded modulation," in Proc. GLOBECOM'00, 2000, pp. 1627-1631,.

[19] G. Bauch, "Concatenation of space-time block codes and "Turbo"-TCM," in Proc. ICC'99, pp. 1999, 1202-1206.

[20] A. Stefanov and T. Duman, "Turbo-coded modulation for systems with transmit and receive antenna diversity over block fading channels: system model, decoding approaches, and practical considerations," IEEE J. Select. Areas Commun., vol. 19, pp. 958-968, May 2001.

[21] Salam Zummo, "Coding and channel estimation for block fading channels", Ph.D. thesis, University of Michigan, Ann Arbor, USA, Jun. 2003.

[22] W. Firmanto, B. Vucetic, and J. Yuan, "Space-time TCM with improved performance on fast fading channels," IEEE Commun. Lett., vol. 5, no. 4, pp. 154-156, Apr. 2001.

[23] S. Zummo and S. Al-Semari, "Space-time coded 8-PSK schemes for rapid rayleigh fading channels," EUR. J. Applied Signal Processing, vol. 5, pp. 482-486, May 2002.

[24] S. Al-Semari and T. Fuja, "I-Q TCM reliable communication over rayleigh fading channel close to the cutoff rate," IEEE Trans. Inform. Theory, vol. 43, pp. 250-262, Jan. 1997.

[25] W. C. Jakes, "Microwave mobile communications", IEEE Press, New Jersy, USA, 1974.

[26] M. Damen, K. Abed-Meraim, and J. Belfiore, "Generalised sphere decoder for asymmetrical space-time communication architecture," Electron. Lett., vol. 36, pp. 166-167, Jan. 2000.

[27] M. Damen, A. Chkeif, and J. Belfiore, "Sphere decoding of space-time codes," in Proc. ISIT'00, 2000, p. 362.

[28] S. H. Jamali and T. Le-Ngoc, "Coded-modulation techniques for fading channels", Kluwer Academic Publisher, Netherlands, 1994.

[29] E. Zehavi and J. Wolf, "On the peformance evaluation of trellis codes," IEEE Trans Inform. Theory, vol. IT-33, pp. 196-202, Mar. 1987. 
[30] E. Biglieri et al. Inroduction to trellis-coded modulation with applications, Macmillan Publishing, New York, USA, 1991.

[31] D. Divsalar and M. Simon, "The design of trellis coded MPSK for fading channels: set partitioning for optimum code design," IEEE Trans. Commun., vol. 36, pp. 1013-1021, September 1988.

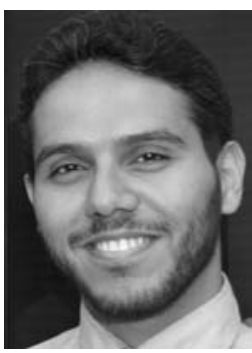

Salam A. Zummo was born in 1976 in Saudi Arabia He received the B.Sc. and M.Sc. degrees in Electrical Engineering from King Fahd University of Petroleum and Minerals (KFUPM), Dhahran, Saudi Arabia, in 1998 and 1999, respectively. He received his PhD degree from the University of Michigan at Ann Arbor, USA, in June 2003. His main research interests include error control coding, diversity, space-time coding, channel estimation, iterative receivers and networking issues for wireless communication systems.

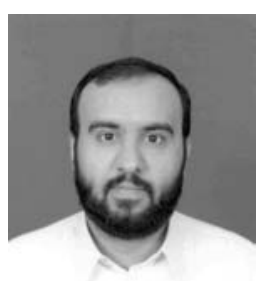

Saud A. Al-Semari received his B.Sc. and M.Sc. degrees in Electrical Engineering from KFUPM in 1991 and 1992, respectively. He received his Ph.D. degree from the University of Maryland at College Park, USA, in December 1995. He is currently an Associate Professor of Electrical Engineering at KFUPM. $\mathrm{He}$ is also the Director of the Information Technology Center at KFUPM. Dr. Al-Semari pursues research in a range of topics related to wireless communication systems and networks including error control coding, diversity, multiple-access and security 